\title{
Using Polymeric Ionic Liquids as an Active Binder in Supercapacitors
}

\author{
Vitor L. Martins, $\oplus^{\mathrm{a}, \mathrm{b}, \mathrm{z}}$ Anthony J. R. Rennie, ${ }^{\mathrm{a}, \mathrm{z}}{ }^{\text {Judith Lesowiec, }}{ }^{\mathrm{a}}$ Roberto M. Torresi, ${ }^{\mathrm{b}}$ \\ and Peter J. Hall ${ }^{\mathrm{a}}$ \\ ${ }^{a}$ Chemical and Biological Engineering, University of Sheffield, Sir Robert Hadfield Building, Sheffield S1 3JD, \\ United Kingdom \\ ${ }^{b}$ Instituto de Química, Universidade de São Paulo, CEP 05513-970 São Paulo, Brazil
}

\begin{abstract}
Electrodes in batteries and supercapacitors generally contain inert binders to maintain their structural integrity during operation but do not participate in the storage of energy. In this paper, we demonstrate that poly ionic liquids can function as structural binders while simultaneously improving the energy storage capability of supercapacitors. Specifically, we show that when the ionic liquid $\mathrm{N}$-butyl- $\mathrm{N}$-methyl pyrrolidinium bis(trifluoromethanesulfonyl)imide is used as electrolyte and poly(diallyldimethylammonium) bis(trifluoromethanesulfonyl)imide is employed as electrode binder the permissible operating voltage of the device is enhanced to $4.0 \mathrm{~V}$. This results in a substantially increased overall specific energy ( $80 \%$ greater) and represents a step toward the development of devices with long cycle lives and high energy densities.

(C) The Author(s) 2017. Published by ECS. This is an open access article distributed under the terms of the Creative Commons Attribution 4.0 License (CC BY, http://creativecommons.org/licenses/by/4.0/), which permits unrestricted reuse of the work in any medium, provided the original work is properly cited. [DOI: 10.1149/2.1411713jes] All rights reserved.

(cc) BY
\end{abstract}

Manuscript submitted September 14, 2017; revised manuscript received October 10, 2017. Published October 27, 2017.

Energy storage devices are continually developing to meet the future demands of clean energy provision, with applications ranging from small portable electronics, to transport and large grid connected systems. ${ }^{1-8}$ From the great variety of available energy storage technologies, electrochemical double-layer capacitors (EDLCs) are associated with high power densities $\left(>10 \mathrm{~kW} \mathrm{~kg}^{-1}\right)$, a high degree of reliability and long cycle life $(>100,000)$, especially when compared to batteries. However, due to their characteristic charge storage mechanism, EDLCs exhibit only a fraction of the energy density achieved by Li-ion batteries. High power densities arise from the electrostatic adsorption of ions at electrode surfaces, however energy density is limited as no significant charge is transferred between the electrodes and electrolyte..$^{2-4,6,8-12}$

Considering this, efforts have been made to increase not only the capacitance of EDLCs but also their energy density, with the ultimate aim of storing as much energy as a battery while retaining the long cycle life of a capacitor. There have been several different approaches toward this objective, from the development of active electrode materials, new device architectures, and the investigation of novel electrolytes including ionic liquids (ILs). ${ }^{1,5,13-15}$

ILs are known for their wide electrochemical stability windows (ESWs). This parameter is critical with respect to the amount of energy that the EDLC can store since $E=\frac{1}{2}\left(C V^{2}\right)$, where $E, C$ and $V$ are energy, capacitance and operating potential, respectively. ${ }^{1,13,15-17}$ For example, the IL $N$-butyl- $N$-methyl pyrrolidinium bis(trifluoromethanesulfonyl)imide $\left(\left[\mathrm{Pyr}_{1,4}\right]\left[\mathrm{Tf}_{2} \mathrm{~N}\right]\right.$, see Figure 1) exhibits a relatively wide ESW and consequently has been extensively studied for EDLC applications. ${ }^{16,18-22}$

EDLC electrodes normally comprise the active material (activated carbon with high surface area), conductive enhancer (carbon black) and a binder (polymer). The polymeric binder is needed to hold all particles together, and normally being inert, does not play any role in the energy storage mechanism. Additionally, the binder is also important in the viscous slurry preparation (mixing the solids components and solvent) that can be cast onto the current collector, in a similar process to those employed in Li-ion batteries manufacturing. It is worth noting that other approaches are also considered in electrode manufacture, as binder-free ${ }^{23-26}$ and self-standing ${ }^{27-29}$ electrodes. Specifically, Miller, Outlaw and Holloway described the growth of vertically oriented graphene directly on the current collectors, greatly improving electronic and ionic resistance. ${ }^{30}$ Eustache and collaborators prepared a binder free 3D micro-supercapacitor for improved areal energy density. ${ }^{31}$

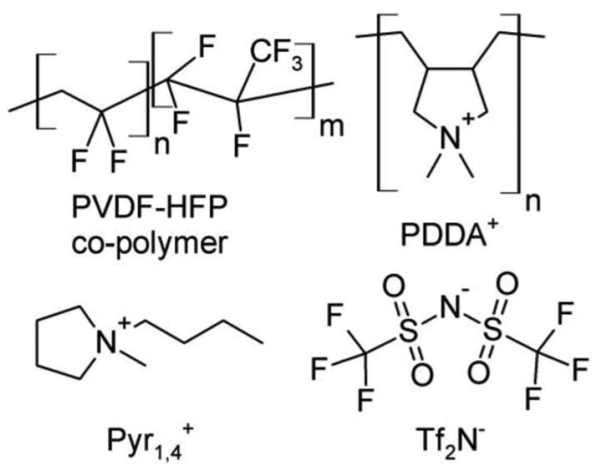

Figure 1. Schematic structure of PVDF-HFP, and [PDDA], [Pyr 1,4$]$ and $\left[\mathrm{Tf}_{2} \mathrm{~N}\right]$ ions.

In this work, we investigated the use of a polymeric IL (poly(IL)) as the electrode binder. The Poly(diallyldimethylammonium) bis(trifluoromethanesulfonyl)imide ([PDDA] $\left[\mathrm{Tf}_{2} \mathrm{~N}\right]$, see Figure 1 ) is a poly(IL) that can produce self-standing membranes when mixed with the IL $\left[\mathrm{Pyr}_{1,4}\right]\left[\mathrm{Tf}_{2} \mathrm{~N}\right]$ in different proportions and have demonstrated interesting results when employed as solid electrolytes in batteries ${ }^{18}$ and EDLCs, ${ }^{19}$ as well as being utilized as a binder material in battery electrodes. ${ }^{32,33}$ Herein, we examine the use of the [PDDA] $\left[\mathrm{Tf}_{2} \mathrm{~N}\right]$ as the binder in EDLC electrode preparation and compare results with cells using a commonly used co-polymer, PVDF-HFP (see Figure 1). Our results show that binder composition plays an important role in defining the electrochemical stability of EDLC; moreover, the use of polymeric ionic liquid as binder permitted a larger maximum operating voltage compared to PVDF-HFP, which has the potential to increase the overall energy density of full devices.

\section{Materials and Methods}

$\left[\mathrm{Pyr}_{1,4}\right]\left[\mathrm{Tf}_{2} \mathrm{~N}\right]$ was purchased from Io-Li-Tec GmbH (>99\%, Germany) and was dried under vigorous stirring and heating $\left(100^{\circ} \mathrm{C}\right)$ for several hours in an argon-filled glove box $\left(\mathrm{H}_{2} \mathrm{O}<0.1 \mathrm{ppm}, \mathrm{O}_{2}<0.1\right.$ $\mathrm{ppm}$ ). Moisture in the dried IL was found to be less than $10 \mathrm{ppm}$ using Karl-Fischer titration (KF899, Metrohm).

$[\mathrm{PDDA}]\left[\mathrm{Tf}_{2} \mathrm{~N}\right]$ was prepared as described elsewhere. ${ }^{18,34}$ Equimolar quantities of PDDA ( $20 \% \mathrm{w} / \mathrm{w}$ solution in water, $M_{W}=100,000$ 200,000, Sigma-Aldrich) and $\operatorname{LiTf}_{2} \mathrm{~N}$ (>99.9\%, Sigma-Aldrich) were mixed in water and the precipitation of $[\mathrm{PDDA}]\left[\mathrm{Tf}_{2} \mathrm{~N}\right]$ as a white 
solid was observed. The solid was filtered, washed with deionized water and dried under vacuum.

EDLC electrodes were prepared by mixing activated carbon $\left(\mathrm{S}_{\mathrm{BET}}\right.$ $=2,120 \mathrm{~m}^{2} \mathrm{~g}^{-1}$ ), conductive carbon black (Super C65) and the binder in 80:10:10 ratio by mass in acetone. The slurries were spread using an adjustable gap paint applicator to a wet film thickness of $100 \mu \mathrm{m}$ on $15 \mu \mathrm{m}$ thick aluminum foil. Sheets were dried at $80^{\circ} \mathrm{C}$ under vacuum overnight prior being punched into individual electrodes with $12 \mathrm{~mm}$ diameter. KynarFlex 2801 (PVDF-HFP), pure $[\mathrm{PDDA}]\left[\mathrm{Tf}_{2} \mathrm{~N}\right]$ and mixtures of [PDDA] $\left[\mathrm{Tf}_{2} \mathrm{~N}\right]:\left[\mathrm{Pyr}_{1,4}\right]\left[\mathrm{Tf}_{2} \mathrm{~N}\right](80: 20$, 60:40 and 40:60 by weight) were used as binder, always maintaining the binder proportion as $10 \%$ weight of the total electrode weight (i.e. $[\mathrm{PDDA}]\left[\mathrm{Tf}_{2} \mathrm{~N}\right]+\left[\mathrm{Pyr}_{1,4}\right]\left[\mathrm{Tf}_{2} \mathrm{~N}\right]=10 \mathrm{wt} \%$, not including the mass of $\left[\mathrm{Pyr}_{1,4}\right]\left[\mathrm{Tf}_{2} \mathrm{~N}\right]$ added as electrolyte).

In order to obtain counter electrodes with at least 20 times the active mass compared with the conventional electrodes, the same composition described above was used with PTFE (Teflon 30-N) as binder in ethanol. This produced self-supporting electrodes with a thickness of $0.5 \mathrm{~mm}$.

Cell assembly was carried out using two electrode button cells (2016) with stainless steel spacers, carbon-based electrodes, and glass fiber filter paper as separator (GF/F, Whatman). The separator was impregnated with the electrolyte, i.e. the IL $\left[\mathrm{Pyr}_{1,4}\right]\left[\mathrm{Tf}_{2} \mathrm{~N}\right]$ in all EDLCs, and the cell was placed under vacuum in the glove box antechamber for at least five minutes to encourage thorough wetting of the electrodes. Cells were crimped inside the glove box.

Scanning electron microscopy (SEM) images were acquired from electrodes prepared as described above using a JEOL JSM-6010LA microscope. Images were acquired with a working distance of ca. $10 \mathrm{~mm}$, and an accelerating voltage of $8 \mathrm{kV}$.

Atomic force microscopy (AFM) images were acquired by dropcasting solutions of polymers or polymer:IL mixtures. The polymer was dissolved in acetone, stirred and drop -cast on microscopy slides. After drying overnight, the films were analyzed using a Dimension Icon instrument (Bruker) in ScanAsyst mode. Images were processed using the Gwyddion software package. ${ }^{35}$

Maximum operating potentials were determined using counter electrodes with a mass at least 20 times greater than the working electrode based on the method of Weingarth et al. ${ }^{36}$ and it is described elsewhere. ${ }^{16}$ Initially, four cyclic voltammograms were performed in the asymmetric cells from open circuit potential (OCP) to $0.5 \mathrm{~V}$ at $5 \mathrm{mV} \mathrm{s}^{-1}$. After that, the window was increased in increments of $0.1 \mathrm{~V}$ to a maximum of $2.0 \mathrm{~V}$. The same procedure was performed using fresh cells from OCP to $-1.0 \mathrm{~V}$, to a maximum of $-3.0 \mathrm{~V}$. $\mathrm{S}$-values were obtained from the last cycle at each electrochemical window, and stability limits identified by a sharp rise in the value of $\mathrm{d}^{2} \mathrm{~S} / \mathrm{dV}^{2}$. Cyclic voltammetry was performed using a Solartron Analytical 1470E Multi-channel potentiostat/galvanostat.

The electrochemical performance of EDLCs were evaluated taking in consideration recommendations from literature. ${ }^{37}$ Cyclic voltammetries using scan rates from 5 to $200 \mathrm{mV} \mathrm{s}^{-1}$ were performed in the same system described above. The specific capacitance, $C_{C V}$, was determined from the charge delivered during the discharge process, $\int i \cdot d t$, the operating potential window, $U$, and the active mass of both electrodes (total mass of activated carbon in cell), $m$, as shown in Equation 1:

$$
C_{C V}=\frac{\int i \cdot d t}{U \cdot m}
$$

Cells were cycled galvanostatically between $0 \mathrm{~V}$ and the operating voltage at different rates from 0.1 and $10 \mathrm{~A} \mathrm{~g} \mathrm{~g}^{-1}$, using a Maccor $4000 \mathrm{M}$ cell test system. Specific capacitance, $C_{G C}$, was determined considering the current, $i$, the slope of the discharge curve after $\mathrm{iR}$ drop, $d V / d t$, and the active mass, $m$, as shown in Equation 2:

$$
C_{G C}=\frac{i}{(d V / d t) \cdot m}
$$

Specific energy, $E_{\text {ave }}$ and specific power, $P_{\text {ave }}$, were determined from the galvanostatic experiments considering the current, $i$, operat- ing voltage, $U$, time of discharge, $t_{d}$, and both electrodes active mass, $m$, using Equations 3 and 4:

$$
\begin{gathered}
E_{\text {ave }}=i \cdot \int \frac{U}{m \cdot 3.6} \cdot d t_{d} \\
P_{\text {ave }}=\frac{E_{\text {ave }} \cdot 3600}{t_{d}}
\end{gathered}
$$

\section{Results and Discussion}

In order to investigate the role of $[\mathrm{PDDA}]\left[\mathrm{Tf}_{2} \mathrm{~N}\right]$ and the mixtures [PDDA] $\left[\mathrm{Tf}_{2} \mathrm{~N}\right]:\left[\mathrm{Pyr}_{1,4}\right]\left[\mathrm{Tf}_{2} \mathrm{~N}\right]$ as an electrode binder, electrodes were prepared using PVDF-HFP, pure [PDDA] $\left[\mathrm{Tf}_{2} \mathrm{~N}\right]$ and mixtures [PDDA] $]\left[\mathrm{Tf}_{2} \mathrm{~N}\right]:\left[\mathrm{Pyr}_{1,4}\right]\left[\mathrm{Tf}_{2} \mathrm{~N}\right]$ as $10 \%$ of total electrode mass. The mixtures poly(IL):IL were incorporated into the electrode formulation process in ratios of 80:20, 60:40 and 40:60 by weight, always representing $10 \%$ of the total electrode mass. Mixtures between both compounds have been shown to be mechanically stable and display reasonable ionic conductivities. ${ }^{18,19,32,34,38,39}$ The $\left[\mathrm{Pyr}_{1,4}\right]\left[\mathrm{Tf}_{2} \mathrm{~N}\right]$ IL was employed as electrolyte in all EDLCs as it has been widely studied in EDLCs, shares anions with the poly(IL) and has a similar chemical structure to the [PDDA] cation.

Physicochemical characterization of the co-polymer, poly(IL) and mixtures of poly(IL):IL were performed using drop-cast films. Fourpoint probe measurements at $25^{\circ} \mathrm{C}$ show that the ionic conductivity of films increases as the proportion of IL increases, from $3.510^{-6} \mathrm{mS}$ $\mathrm{cm}^{-1}$ for pure [PDDA] $\left[\mathrm{Tf}_{2} \mathrm{~N}\right.$ ] to $0.31 \mathrm{mS} \mathrm{cm}^{-1}$ for the $40: 60$ mixture, which is in good agreement with previous work, ${ }^{19}$ whereas the neat IL has a conductivity of $2.7 \mathrm{mS} \mathrm{cm} \mathrm{cm}^{-1}$ (see Figure S1, in Supplementary Information (SI)). The pure [PDDA] $\left[\mathrm{Tf}_{2} \mathrm{~N}\right]$ and IL also display high thermal stability, with decomposition temperatures of 435 and $455^{\circ} \mathrm{C}$, respectively. The three mixtures display similar decomposition temperatures to the poly(IL), and exhibit one step decomposition processes, as suggested by Figure S2a and S2b (SI). Differential scanning calorimetry analyses presented in Figure S2c (SI) show that the mixtures form a composite with significantly different thermal properties when compared to the pure IL and do not undergo clear phase transitions over the temperature range studied.

Electrodes containing activated carbon, carbon black and the binder were cast on aluminum foil using an adjustable paint applicator. Figures $2 \mathrm{a}-2 \mathrm{c}$ show SEM images of electrodes containing copolymer, poly(IL) and the mixture poly(IL):IL 40:60, respectively. It can be seen that there is little difference in appearance, with the primary particles of active material roughly $5 \mu \mathrm{m}$ in size being uniformly coated with binder. It is worth noting that the presence of the IL during the drying of the electrode is likely to influence the morphology of the resulting polymer by inhibiting shrinkage or by acting as a plasticizer, as can be seen in Atomic Force Microscopy (AFM) images in Figure S3, SI. However, there was no significant difference in the physical appearance or mechanical stability of the electrodes prepared using IL to those without. The AFM images show that all films present subtly different morphologies. PVDF-HFP seems to consist of interconnected chains and scans in smaller areas shows that the co-polymer exhibits a granular texture. [PDDA] $\left[\mathrm{Tf}_{2} \mathrm{~N}\right]$ displays a porous morphology, which may be due to the drying process. Scans over smaller regions show a less granular morphology than that of the co-polymer. Also, as the amount of IL increases in the poly(IL):IL mixtures the pore density is seen to decrease. The mixture with highest content of IL has a completely different morphology than the $[\mathrm{PDDA}]\left[\mathrm{Tf}_{2} \mathrm{~N}\right]$ alone. These different morphologies are also evidenced by apparent changes in their physical properties since an increase in pliability was found to accompany an increase in IL fraction.

Electrodes were also cast onto the current collector using different wet film thicknesses. Figure $2 d$ shows that electrode thickness linearly increases with active mass, independent of the binder employed. Overall, the electrodes have similar density over the obtained thicknesses, as confirmed in Figure 2e. Co-polymer, poly(IL) and mixture 

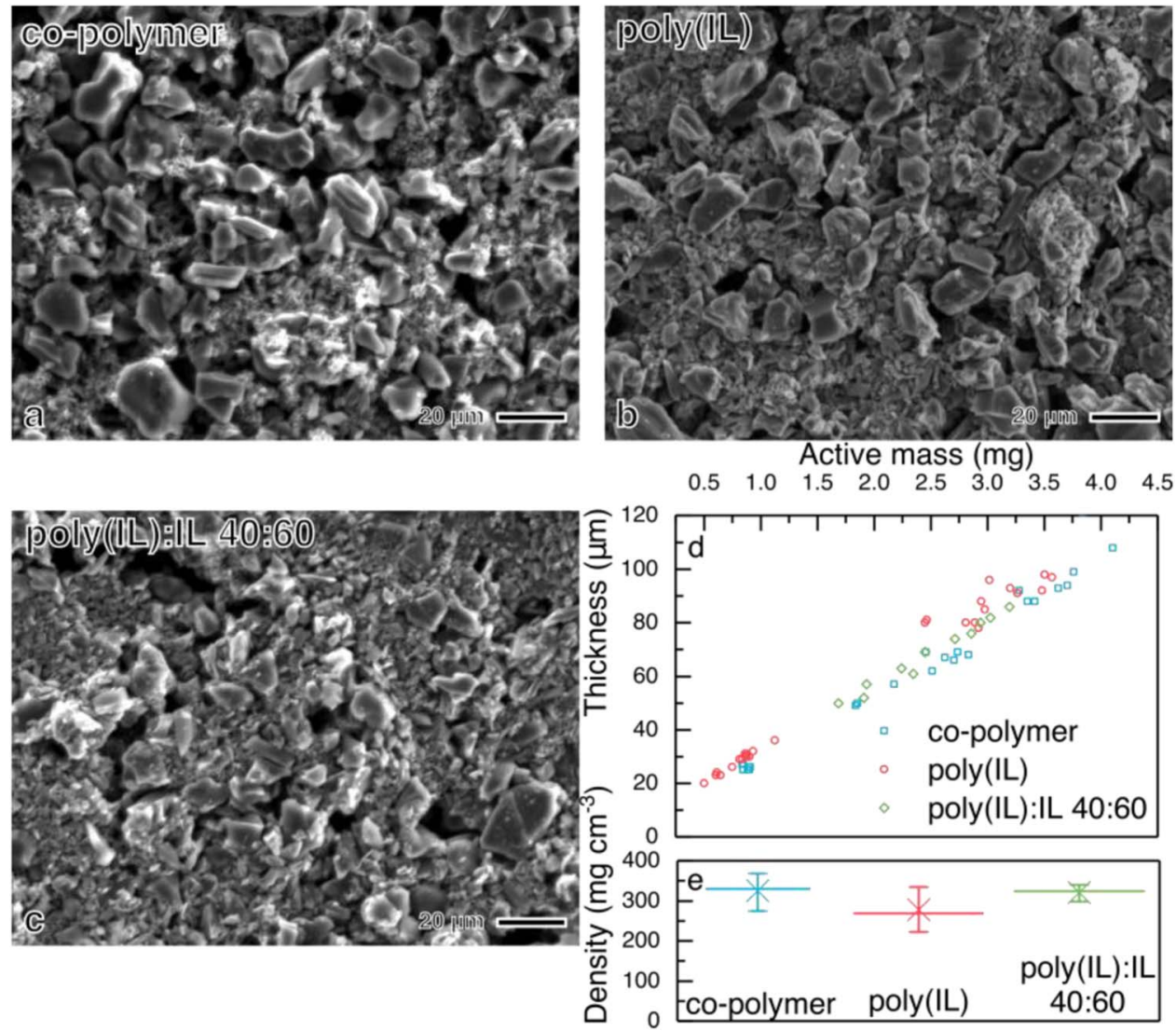

Figure 2. SEM images of EDLC electrodes prepared with (a) co-polymer PVDF-HFP, (b) poly(IL) [PDDA][Tf 2 N] and (c) a mixture of poly(IL):IL [PDDA] $\left.] \mathrm{Tf}_{2} \mathrm{~N}\right]:\left[\mathrm{Pyr}_{1,4}\right]\left[\mathrm{Tf}_{2} \mathrm{~N}\right](40: 60, \mathrm{~m} / \mathrm{m})$, respectively. (d) Relationships between electrode thickness, mass and (e) average density.

poly(IL):IL (60:40) electrodes possessed densities of $325( \pm 25), 277$ $( \pm 33)$ and $321( \pm 13) \mathrm{mg} \mathrm{cm}^{-3}$, respectively. It is worth noting that the adhesion with the current collector in thick electrodes was relatively poor, producing electrodes that detach and/or break during production. This resulted in an inadequate level of reproducibility in EDLCs with thicker electrodes and for this reason, the EDLCs studied here have mass loadings from 1.0 to $2.5 \mathrm{mg} \mathrm{cm}^{-2}$.

In order to maximize energy density, it is necessary to determine the largest stable operating potential of the EDLC electrodes/electrolytes. The ESW of $\left[\mathrm{Pyr}_{1,4}\right]\left[\mathrm{Tf}_{2} \mathrm{~N}\right]$ is influenced by many factors, especially the electrode materials employed. The maximum operating potential was determined by adapting the approach reported by Weingarth et al. ${ }^{36}$ using a coin cell arrangement. ${ }^{16}$ Figure 3 a shows the "S-value", which is a measure of the relative coulombic efficiency of cells, over different electrochemical windows for the co-polymer, poly(IL) and mixture poly(IL):IL 40:60.

Scanning to positive potentials shows that the electrodes containing PVDF-HFP are more stable than those using poly(IL) in binder formulation. However, scanning to negative potentials shows a much greater stability for the electrode containing poly(IL). The positive limits differ by only $0.1 \mathrm{~V}$, whereas negative limits of $-2.5 \mathrm{~V}$ and $-2.0 \mathrm{~V}$ were identified for the poly(IL) based binders and co-polymer respectively. Combining both potential limits results in an operating potential of $4.0 \mathrm{~V}$ for cells using $[\mathrm{PDDA}]\left[\mathrm{Tf}_{2} \mathrm{~N}\right]$ which is significantly greater than the value of $3.6 \mathrm{~V}$ determined for the co-polymer. The observed difference in electrochemical stability in the negative scan indicates that interactions between the binder and IL electrolyte $\left[\mathrm{Pyr}_{1,4}\right]\left[\mathrm{Tf}_{2} \mathrm{~N}\right]$ play an important role in electrolyte decomposition, possibly delaying the onset of decomposition in certain potential regions. In the case of [PDDA] $\left[\mathrm{Tf}_{2} \mathrm{~N}\right]$ based binders, ie. pure poly(IL) and mixture poly(IL):IL, it is reasonable to assume that a substantial degree of coordination between the binder and electrolyte occurs as they share the same anion. It is worth noting that the specific capacitance in the negative scan is considerably higher for electrode containing $[\mathrm{PDDA}]\left[\mathrm{Tf}_{2} \mathrm{~N}\right]$ in the binder formulation than for the copolymer electrode (ca. 120 and $90 \mathrm{~F} \mathrm{~g}^{-1}$ ). As [PDDA] $\left[\mathrm{Tf}_{2} \mathrm{~N}\right.$ ] presents a fixed cation and a mobile anion, charge compensation by the cation in the negative scan may be augmented by the mobility of the binder anion. However, at positive potentials the cation is immobile and unable to contribute toward the charge compensation process. Consequently, the electrolyte is required to balance the charge, and in this case the poly(IL) based EDLC presents a similar specific capacitance to the co-polymer-EDLC. It should be noted that since the operating potential windows are asymmetric different electrode mass loadings are necessary to avoid electrolyte degradation. ${ }^{2}$ The potential limits illustrated in Figure $3 \mathrm{a}$ were used to define the electrode mass ratios for these systems; therefore, the ratio of positive to negative electrode mass for the co-polymer-EDLC and poly(IL)-EDLCs were 1.25 and 1.67, respectively. Considering the coulombic charge involve in the discharge process of each determination, the mass ratio would be 1.05 , 2.04 and 1.95 for co-polymer, poly(IL) and the mixture poly(IL):IL 40:60, respectively.

Figure $3 \mathrm{~b}$ presents cyclic voltammograms at $5 \mathrm{mV} \mathrm{s}^{-1}$ from $0 \mathrm{~V}$ to the defined operating potential for EDLCs containing co-polymer and poly(IL) in binder formulation (intermediary mixture of poly(IL)-IL are showed in Figure S4 in SI). All systems display typical EDLC profiles, with a quasi-rectangular shape and no peaks that would indicate the occurrence of faradaic reactions. The improved stability of cells using [PDDA] $\left[\mathrm{Tf}_{2} \mathrm{~N}\right]$ as binder is further evidenced in Figure $3 \mathrm{~b}$ as the current response of the co-polymer cell can be seen to rise as the potential approaches $3.5 \mathrm{~V}$. Cells using co-polymer and poly(IL) present similar specific capacitances at $5 \mathrm{mV} \mathrm{s}^{-1}$, ca. $23 \mathrm{~F} \mathrm{~g}^{-1}$. However, a notable difference between the electrodes prepared using poly(IL):IL mixtures and those with co-polymer and pure poly(IL) can be seen in 

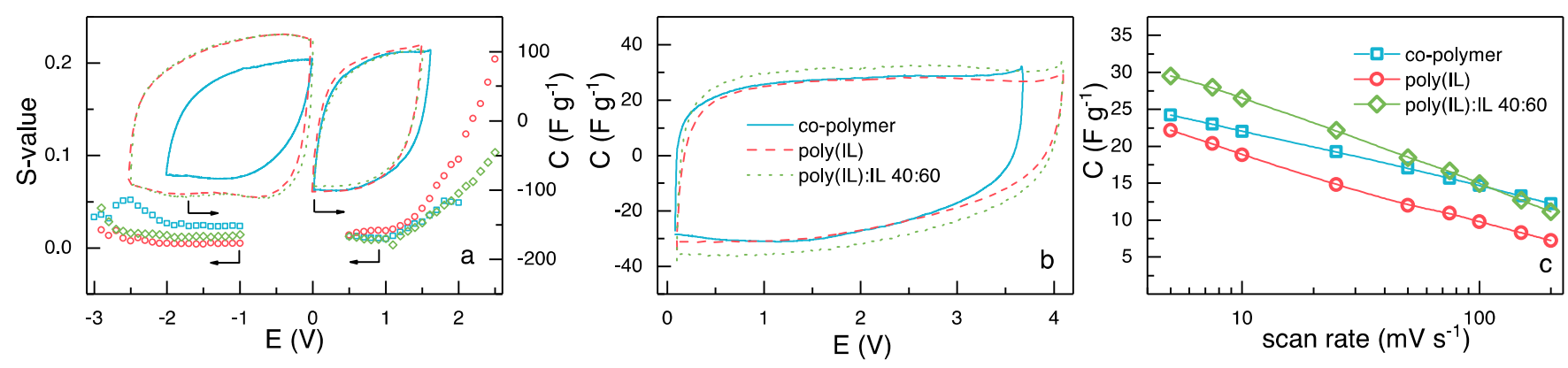

Figure 3. (a) Determination of operating potential by cyclic voltammetry at $5 \mathrm{mV} \mathrm{s}^{-1}$, using a counter-electrode with at least 20 times the mass of the working electrode. Left axis shows "S-values" of the third cycle of cells in the anodic and cathodic limit determination of electrodes containing co-polymer (blue square), poly(IL) (red circle) and poly(IL):IL mixture (40:60, m/m) (green diamond) as binder. Right axis shows cyclic voltammetry at the determined operating potential of electrodes containing co-polymer (blue full line), poly(IL) (red dashed line) and poly(IL):IL mixture (green dotted line) as binder. (b) Cyclic voltammetry of EDLCs at determined voltages at $5 \mathrm{mV} \mathrm{s}^{-1}$. (c) Specific capacitance of the EDLCs containing the different binders at different scan rates.

the values of specific capacitance. At a rate of $5 \mathrm{mV} \mathrm{s}^{-1}$, the specific capacitance of cells using a poly(IL):IL 80:20 mixture was $25.3 \mathrm{~F}$ $\mathrm{g}^{-1}$, which increases to $29.5 \mathrm{~F} \mathrm{~g}^{-1}$ for that using a 40:60 mixture; this represents a substantial increase in specific capacitance when compared with those using no IL in electrolyte manufacture $\left(22.2 \mathrm{~F} \mathrm{~g}^{-1}\right)$. Figure $3 \mathrm{c}$ shows that as the scan rate is increased the co-polymer retains a greater proportion of the capacitance determined at $5 \mathrm{mV}$ $\mathrm{s}^{-1}$. This indicates a greater degree of resistive losses associated with poly(IL) electrodes. But the poly(IL):IL 40:60 EDLC even showing a low retention as the scan rate increases still presents a similar specific capacitance to that obtained with the co-polymer EDLC. The high specific capacitance at slow scan rate but the low retention suggests that there is a higher degree of resistance associated with electrodes using $[\mathrm{PDDA}]\left[\mathrm{Tf}_{2} \mathrm{~N}\right]$ and that any benefits arising from ion conduction in the binder are only realized at relatively low rates. The inclusion of the IL in electrode preparation may enhance the specific capacitance in several ways; mixing with acetone may facilitate electrolyte access to smaller pores of the activated carbon, or the IL may interact with $[\mathrm{PDDA}]\left[\mathrm{Tf}_{2} \mathrm{~N}\right]$ to alter the drying behavior of the polymer, creating different morphologies, as suggested by AFM.

A wider range of charge-discharge rates was explored using galvanostatic cycling at rates between 0.1 and $10 \mathrm{~A} \mathrm{~g}^{-1}$ and the results are given in Figure 4a. As with cyclic voltammetry, the specific capacitance of the poly(IL)-EDLC is similar to the co-polymer-EDLC at low rates. However, as the current increases, the specific capacitance of the $[\mathrm{PDDA}]\left[\mathrm{Tf}_{2} \mathrm{~N}\right]$ cells drops considerably. Similar rate dependent behavior to that observed using cyclic voltammetry is also seen in Figure 4a when the poly(IL):IL mixture was used in electrode preparation. The mixtures of poly(IL):IL present greater specific capacitance at low rates, however at rates greater than $5 \mathrm{~A} \mathrm{~g}^{-1}$, the co-polymer-EDLCs outperforms all mixtures. At $10 \mathrm{~A} \mathrm{~g}^{-1}$, all of EDLCs containing [PDDA] $\left[\mathrm{Tf}_{2} \mathrm{~N}\right]$ present an insignificant amount of charge retention, while the co-polymer-EDLC maintains ca. $33 \%$ of the capacitance determined at the lowest rate.

The EDLCs were also investigated by electrochemical impedance spectroscopy (EIS) and the resulting Nyquist plots are given in Figures $4 \mathrm{~b}-4 \mathrm{c}$, and Figure S6 in SI for intermediate poly(IL):IL mixtures. The spectra are of similar shape, with a semi-circle at high frequencies and linearity at low frequencies, as already observed for EDLCs containing IL electrolytes. ${ }^{40}$ The diameter of the semi-circle seen at high frequencies (internal resistance, $R_{i}$ ) is more than five times larger for the poly(IL)-EDLC than for the co-polymer-EDLC (71.1 vs $12.5 \Omega$ ). The poly(IL):IL mixtures exhibits a slight decrease in the $\mathrm{R}_{\mathrm{i}}$ as the fraction of IL increases. The $R_{i}$ is related with the overall resistance in the device, taking in account the interconnectivity among activated particles and additive particles, how they are packed and make contact with the current collector, in addition to the resistance toward the movement of ions inside pores. ${ }^{41}$ One of the reasons for the higher resistance may arise from the reduced mobility of ions due to their interactions with the binder, or due to poorer interconnectivity between particles. Ion migration is likely to be affected by co-ordination between the electrolyte and binder because they share the same anion. Electrostatic interactions between the electrolyte anions and the covalently bound cations of the binder would hinder ion migration and therefore be observed as an increased resistance. As the ratio of IL in the binder increases, the diameter of the semi-circles in the spectra are seen to decrease which again may be attributed to the relative fraction of [PDDA] $\left[\mathrm{Tf}_{2} \mathrm{~N}\right]$ present in the electrode. It is also possible that the diminished resistance is due to the increasing quantity of IL present in the electrode production process. It is worth noting that as the frequency decreases, the phase angle approaches $90^{\circ}$, as expected for a capacitor. In-situ and in-operando techniques could clarify the raise of such bigger resistance in poly(IL) electrodes. In-operando NMR and quartz-crystal microbalance could potentially elucidate
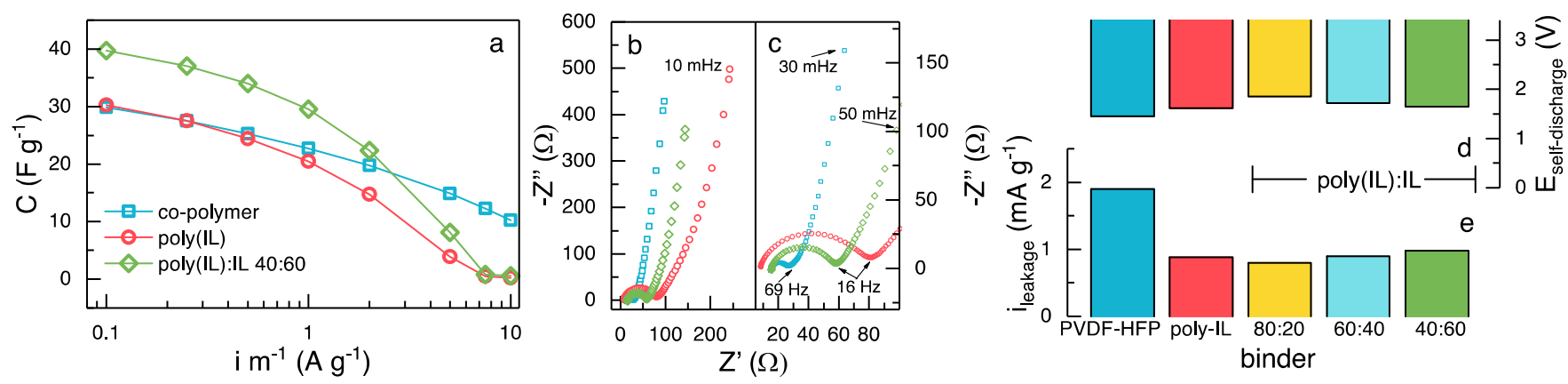

Figure 4. (a) Specific capacitance of the EDLCs with co-polymer (blue square), poly(IL) (red circle) and poly(IL):IL mixture 40:60 (green diamond) as binders determined using galvanostatic charge-discharge cycling at different rates, from 0.1 to $10 \mathrm{~A} \mathrm{~g}^{-1}$. (b) Nyquist plots from EIS of EDLCs and (c) Magnified high frequency region. (d) Self-discharge voltage and (e) leakage current of EDLCs containing different binders. 

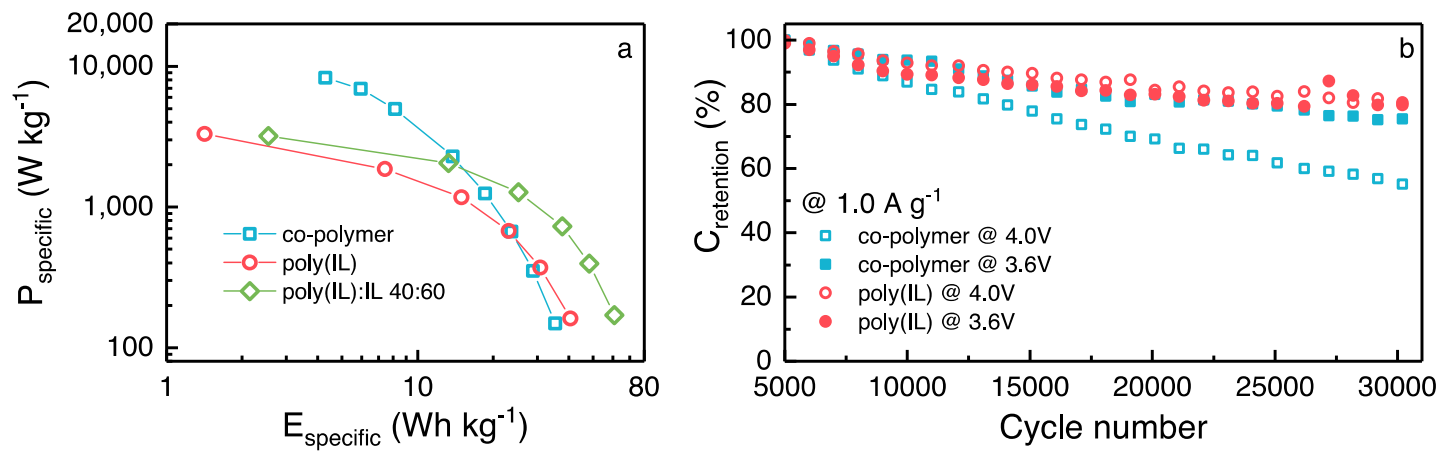

Figure 5. (a) Ragone plot of the EDLCs containing co-polymer (blue square), poly(IL) (red circle) and poly(IL):IL mixture (green diamond) obtained from the galvanostatic charge-discharge at different rates. (b) Long-term stability determination for the co-polymer-EDLC (blue square) and the poly(IL)-EDLC (red circle) at $1.0 \mathrm{~A} \mathrm{~g}^{-1}$ from 0 to $3.6 \mathrm{~V}$ (closed symbols) and $4.0 \mathrm{~V}$ (open symbols).

the charge balance process during high frequencies sweep and determined the role played by the polymer in such process. ${ }^{42,43}$ The high $\mathrm{R}_{\mathrm{i}}$ associated with poly(IL) based electrodes could suggest a higher self-discharge and leakage current than the co-polymer electrodes, however Figures $4 d-4 e$ demonstrate otherwise. Figure $4 d$ shows the self-discharge voltage $\left(\mathrm{E}_{\text {self-discharge }}\right)$ and Figure $4 \mathrm{e}$ the leakage current $\left(i_{\text {leakage }}\right)$ determined for each of the studied EDLCs. For $E_{\text {self-discharge }}$ determination, all EDLCs were held at the same voltage (3.6 V, for a better comparison) for 1 hour. After 72 hours, the co-polymer-EDLC presented the lowest $\mathrm{E}_{\text {self-discharge, }}$ indicating that the EDLCs containing poly(IL) in the binder formulation retain charge for longer than the co-polymer EDLC; voltage profiles are presented in Figure S5(a) in SI. On the other hand, $\mathrm{i}_{\text {leakage }}$ was determined by holding the same charging voltage $(3.6 \mathrm{~V})$ for 72 hours, and the current measured at the end of hold time is illustrated in Figure 4e. In order to maintain $3.6 \mathrm{~V}$, the co-polymer requires more than twice the current required for the EDLCs containing poly(IL) in binder formulations. The profiles related to the determination of $i_{\text {leakage }}$ are given in Figure S5(b) in SI.

The specific power and energy derived from the galvanostatic discharge steps are expressed in the form of a Ragone plot in Figure 5a. It is clear that the higher operating potential of cells using [PDDA] $\left[\mathrm{Tf}_{2} \mathrm{~N}\right]$ substantially increases their specific energy. Most strikingly, it is seen that cells using a mixture of poly(IL):IL (40:60) in electrode preparation can store nearly twice the amount of energy as that using a co-polymer binder. However, this is only the case at lower rates and it is clear that this binder is associated with poorer high rate performance in comparison to the co-polymer. The long-term cycling at $1.0 \mathrm{~A} \mathrm{~g}^{-1}$ shown in Figure $5 \mathrm{~b}$ reveals that the poly(IL)-EDLC operating at $4.0 \mathrm{~V}$ has a long-term stability very similar to the copolymer-EDLC operating at $3.6 \mathrm{~V}$, supporting the results from Figure $3 \mathrm{a}$ and confirming that EDLCs assembled using the [PDDA][Tf $\left.{ }_{2} \mathrm{~N}\right]$ can operate at greater potentials than the co-polymer. It is worth noting that the poly(IL)-EDLCs operating at 4.0 and $3.6 \mathrm{~V}$ display similar stability for long-term cycling, indicating that there is little degradation occurring over this increased window and that co-polymer-EDLC shows a much quicker fade in capacitance.

\section{Conclusions}

In summary, using the poly(IL) $[\mathrm{PDDA}]\left[\mathrm{Tf}_{2} \mathrm{~N}\right]$ as the binder in the electrode preparation process increases the electrochemical stability of the electrolyte and therefore the operating potential of the device. EDLCs assembled with PVDF-HFP or [PDDA] $\left[\mathrm{Tf}_{2} \mathrm{~N}\right]$ possess similar specific capacitances at low charge/discharge rates, however the interactions between ions of the IL and [PDDA] $]\left[\mathrm{Tf}_{2} \mathrm{~N}\right]$ that enhance electrochemical stability also appear to inhibit performance at high rates. Incorporating IL in poly(IL) based electrode preparation increased specific capacitance. The substantial increase in the electrochemical stability of the device has a significant effect on the resultant specific energy, with this characteristic being almost doubled at low discharge rates. EDLCs containing [PDDA] $\left[\mathrm{Tf}_{2} \mathrm{~N}\right]$ as binder presents similar long-term stability at a potential of $4.0 \mathrm{~V}$ to those containing the co-polymer at a potential of $3.6 \mathrm{~V}$, indicating that $[\mathrm{PDDA}]\left[\mathrm{Tf}_{2} \mathrm{~N}\right]$ performs well as a structural binder during extended cycling. This represents a step toward devices with long cycle lives and high energy densities. Furthermore, we believe that poly(ILs) have the potential to be used as electrode binders in many applications, and can be considered as active components in supercapacitor and battery production.

\section{Acknowledgments}

The authors acknowledge EPSRC (EP/K021192/1) and FAPESP (15/26308-7, 13/22748-7 and 14/14690-1) for funding. The authors also acknowledge EPSRC (EP/K001329/1 (4CU)) for the SEM facility.

\section{ORCID}

Vitor L. Martins (D) https://orcid.org/0000-0002-8824-7328

Anthony J. R. Rennie (1) https://orcid.org/0000-0002-4254-8352

\section{References}

1. D. R. MacFarlane et al., Energy Environ. Sci., 7, 232 (2014)

2. M. Mastragostino and F. Soavi, J. Power Sources, 174, 89 (2007).

3. E. Frackowiak and F. Béguin, Carbon N. Y., 39, 937 (2001).

4. J. R. Miller and P. Simon, Science, 321, 651 (2008).

5. M. Armand, F. Endres, D. R. MacFarlane, H. Ohno, and B. Scrosati, Nat. Mater, 8 , $621(2009)$

6. P. Simon and Y. Gogotsi, Nat. Mater., 7, 845 (2008).

7. M. Winter and R. J. Brodd, Chem. Rev., 104, 4245 (2004)

8. P. J. Hall et al., Energy Environ. Sci., 3, 1238 (2010).

9. F. Béguin, V. Presser, A. Balducci, and E. Frackowiak, Adv. Mater., 26, 2219 (2014)

10. C. Merlet et al., Nat. Mater., 11, 306 (2012).

11. J. R. Miller and A. F. Burke, Electrochem. Soc. Interface, 17, 53 (2008).

12. D. R. MacFarlane et al., Nat. Rev. Mater., 1, 15005 (2016).

13. M. Galiński et al., Electrochim. Acta, 51, 5567 (2006).

14. A. Lewandowski and A. Świderska-Mocek, J. Power Sources, 194, 601 (2009).

15. A. Brandt, S. Pohlmann, A. Varzi, A. Balducci, and S. Passerini, MRS Bull., 38, 554 (2013).

16. A. J. R. Rennie, V. L. Martins, R. M. Torresi, and P. J. Hall, J. Phys. Chem. C, 119, 23865 (2015).

17. A. Noofeli, P. J. Hall, and A. J. R. Rennie, Faraday Discuss., 172, 163 (2014)

18. A.-L. Pont, R. Marcilla, I. De Meatza, H. Grande, and D. Mecerreyes, J. Power Sources, 188, 558 (2009).

19. G. Ayalneh Tiruye, D. Muñoz-Torrero, J. Palma, M. Anderson, and R. Marcilla, J. Power Sources, 279, 472 (2015).

20. S. Pohlmann et al., J. Power Sources, 273, 931 (2015).

21. A. Balducci et al., J. Power Sources, 165, 922 (2007).

22. A. Krause and A. Balducci, Electrochem. Commun., 13, 814 (2011).

23. E. D. Walsh et al., ACS Appl. Mater. Interfaces, 8, 29478 (2016).

24. G. Luo et al., Appl. Surf. Sci., 366, 46 (2016).

25. N. Díez, C. Botas, E. Goikolea, and D. Carriazo, J. Mater. Sci., 52, 11191 (2017).

26. J. Yan et al., Adv. Funct. Mater, 27, 1701264 (2017).

27. Y. Liu et al., Sci. Rep., 5, 17045 (2015).

28. M. Zhu et al., Adv. Energy Mater., 6, 1600969 (2016).

29. Z. Fan et al., ACS Appl. Mater. Interfaces, 9, 21763 (2017). 
30. J. R. Miller, R. A. Outlaw, and B. C. Holloway, Science, 329, 1637 (2010).

31. E. Eustache et al., Adv. Mater. Technol., 2, 1700126 (2017).

32. J.-S. Lee, K. Sakaushi, M. Antonietti, and J. Yuan, RSC Adv., 5, 85517 (2015).

33. J. von Zamory, M. Bedu, S. Fantini, S. Passerini, and E. Paillard, J. Power Sources, 240, 745 (2013).

34. T. M. Benedetti and R. M. Torresi, Langmuir, 29, 15589 (2013).

35. D. Nečas and P. Klapetek, Open Phys. 10, 181 (2012).

36. D. Weingarth, H. Noh, A. Foelske-Schmitz, A. Wokaun, and R. Kötz, Electrochim. Acta, 103, 119 (2013).
37. A. Balducci, D. Belanger, T. Brousse, J. W. Long, and W. Sugimoto, J. Electrochem. Soc., 164, A1487 (2017)

38. A. Balducci et al., J. Power Sources, 196, 9719 (2011).

39. G. B. Appetecchi et al., J. Power Sources, 195, 3668 (2010).

40. A. J. R. Rennie, N. Sanchez-Ramirez, R. M. Torresi, and P. J. Hall, J. Phys. Chem. Lett., 4, 2970 (2013).

41. P. L. Taberna, C. Portet, and P. Simon, Appl. Phys. A, 82, 639 (2006).

42. N. Shpigel et al., ACS Energy Lett., 2, 1407 (2017).

43. A. C. Forse et al., Nat. Energy, 2, 16216 (2017). 